\title{
Research on the Influence of Anti-fatigue Effect and Movement Ability of Blueberry Polysaccharides on Aged Mice
}

\author{
Bai Bo* \\ Harbin University of Science and Technology, Harbin, China
}

\begin{abstract}
The article introduces functions as well as extraction and purification of polysaccharides, and the effect of blueberry polysaccharides (BPs) on movement endurance, anti-fatigue capacity and related physiological indexes in aged mice induced by D-galactose is going to be investigated. The method of water extract-alcohol precipitation is used in the experiment to extract polysaccharides from blueberries, and in-vitro oxidation resistance and bacteriostasis of polysaccharides are studied after preliminary purification so as to provide a reference for the production and application of blueberry polysaccharides. Mice are randomly divided into a normal control group, an aged model group, a BP low-dose group, a BP medium-dose group, and a BP high-dose group. After the administration of BPs for 20 consecutive days, the exhaustive swimming time and fatigue indexes are determined. The results show that BPs could prolong the exhaustive swimming time, decrease the content of BUN, BLA and MDA, and obviously increase the activity of HG, MG, SOD and LDH. To conclude, BPs have good anti-fatigue effect. This study may provide theoretical evidence for the development of antifatigue drugs from BPs.
\end{abstract}

Keywords: Anti-fatigue effect, blueberry polysaccharides, exhaustive swimming, extraction, purification D-galactose.

\section{INTRODUCTION}

The blueberry is a wild plant native to North America, Scotland, and Russia and has high cold resistance. Blueberry fruits not only are moderate in sweet and sour and good in flavor, but also contain a lot of ingredients that are good for human health, such as rich vitamins, dietary fiber, protein and other nutrients including antioxidants, tannic acid, folic acid, antibacterial ingredients, etc. Therefore, it is often known as the "king of berries".

Research abroad on active ingredients in blueberries has mainly focused on anthocyanins, and various physiological functions of anthocyanin extract and influences of processing on its antioxidant capacity have been studied [1]. The functional property of the flavonoids in blueberries is also studied. However, there have been few studies on the activity of blueberry poly-saccharides. In the experiment, the polysaccharides are extracted from blueberries with the water extract-alcohol precipitation method and then purified preliminarily, and the in-vitro oxidation resistance and bacteriostasis of polysaccharides are studied, so that a reference can be provided for the production and application of blueberry polysaccharides.

\section{EXTRACTION AND PURIFICATION OF POLY- SACCHARIDES}

\subsection{Extraction of Polysaccharides}

Extraction and separation of polysaccharides including processes as follows: degreasing, solvent extraction, deproteinization, decoloration, etc.

*Address correspondence to this author at the Harbin University of Science and Technology, Harbin, China; Tel: 18986139113 ;

E-mail: hunter2011@foxmail.com
Degreasing: firstly, materials for polysaccharide extraction are degreased in solvents including general or dilute salt water, dilute alkali and dilute acid under different temperatures.

Deproteinization: polysaccharides after organic solvent precipitation often contain more protein required to be removed [2].

Decolorization: coarse polysaccharides often contain some pigments (free combination dyes and pigments), and different methods are adopted according to different properties.

\subsection{Purification of Polysaccharides}

Methods of polysaccharide purification usually comprise the following several kinds:

(1) Division precipitation method: division of the precipitation is performed based on the different solubility properties of polysaccharides in different concentrations of alcohol and ketones, (such as methanol, ethanol and acetone). This method is applicable to the separation of the polysaccharides with various solubility properties [3].

(2) Salt fractionation method: Different polysaccharides have different solubilities in salt with different concentrations. Common salting out agents comprise sodium chloride, potassium chloride and ammonium sulfate etc., wherein sulfuric acid amine is the best.

Metal complex method: polysaccharides can form complex ions and precipitate with copper, barium, calcium, lead sheath. 


\section{MATERIALS AND METHODS}

\subsection{Materials and Reagents}

Firstly, fresh blueberries without freezing injury were bought at the fruit supermarket. Secondly, D-galactose was offered by Sigma-Aldrich companies in the United States. Thirdly, blood urea nitrogen (BUN) kit, blood lactic acid (BLA) kit, liver glycogen, hepatic glycogen (HG) kit, muscle glycogen (MG) kit, malondialdehyde (MDA) kit, superoxide dismutase (SOD) kit, and lactate dehydrogenase (LDH) kit, were offered by Nanjing Jiancheng Biological Engineering Research Institute. Fourthly, altogether 50 Kunming mice, which were at the SPF level and 20-22 g heavy and comprise half female and half male mice, were selected. Other reagents were national analytical reagents.

\subsection{Instrument and Equipment}

DHG-9240A thermostatic drum wind drying oven, DK8D three-hole thermostatic water tank from Shanghai Qixin scientific instrument co. LTD, ultrasonic cleaning machine from Ningbo xingzhi biological technology co. LTD, refrigerated centrifuge from Beckman company in the United States and UV-2450 ultraviolet-visible spectrophotometer from Shimadzu.

\subsection{Preparation of Blueberry Polysaccharides}

$120 \mathrm{~g}$ blueberry fruits were weighed, pulped after quickfreeze at $-80^{\circ} \mathrm{C}$ for $10 \mathrm{~min}$ after being cleaned, a small amount of distilled water is added, and a microwave dissolver is used for extraction. Filtrate is centrifuged at $5000 \mathrm{r} / \mathrm{min}$ for $15 \mathrm{~min}$ after suction filtration. A small amount of water is added, pulp is dissolved after repeated extraction twice, the extracted supernatant fluids are combined and uniformly mixed, and water in the solution is completely evaporated through rotary evaporation; $95 \%$ of ethanol, $100 \%$ of ethanol and acetone are added for processing the solute respectively; the processed mixture is placed at $4^{\circ} \mathrm{C}$ for $24 \mathrm{~h}$ and centrifuged at $3000 \mathrm{r} / \mathrm{min}$ for 10 min. Protein is removed with a Sevag method, and the coarse blueberry polysaccharides were obtained after the precipitate is freeze-dried [4].

\subsection{Conditions and Grouping of Experimental Animals}

Before the trial, 50 mice were fed with basic feed for 1 week, and then they were randomly divided into 5 groups by weight (half male and half female). The mice were divided into the normal control group, the model control group, the BPs low-dose group, the BPs medium-dose group and the BPs high-dose group respectively. The normal control group was intraperitoneally injected with $100 \mathrm{mg} /(\mathrm{kg} \cdot \mathrm{d}$ ) (by weight, similarly hereinafter) of physiological saline, the rest of four groups were intraperitoneally injected with $100 \mathrm{mg} /$ (Kg.d) of D-galactose, and the injection cycle is 60 days. On the $41^{\text {th }}$ days, the normal control group is administered intragastrically with distilled water, the model control group is administered intragastrically with distilled water, while the low-dose, medium-dose and high-dose groups of BPs are administered intragastrically with 100,200 and $400 \mathrm{mg} /$ (Kg.d) of D-galactose and the administration lasts for 20 days. All the groups are fed with basic feed and allowed to drink water freely.

\subsection{Exhaustive Swimming Time of Mice}

$30 \mathrm{~min}$ after the last treatment, the tail of each mouse is loaded with a lead wire $5 \%$ the weight of the mouse, and the degree of tightness of fixation shall be appropriate. The mice are put in 10 thermostatic water tanks respectively with the water depth being water depth $(20+/-5) \mathrm{cm}$ and the water temperature being $25^{\circ} \mathrm{C}$ for exhaustive swimming experiment. Record the mice swimming time with a stopwatch, and the time from the beginning to the time when the mice sink in water without emerging from the water in $10 \mathrm{~s}$ is adopted as the exhaustive swimming time/min. Some mice floating in the water do not swim, which will make the swimming time significantly prolonged, therefore, in order to get reliable results, each mouse must be poked with a stick to swim continuously.

\subsection{Preparation and Testing of Test Sample}

After exhaustive swimming experiment, pull eyeball and draw blood, centrifuge the blood at $4000 \mathrm{r} / \mathrm{min}$ for $10 \mathrm{~min}$, and take supernatant for standby application. All treatment is completed at a temperature of $4^{\circ} \mathrm{C}$. The indexes are tested by BUN, BLA, HG, MG, MDA, SOD and LDH kits in combination with the ultraviolet-visible spectrophotometer.

\section{RESULTS AND ANALYSIS}

The exercise endurance of mice is obtained through the exhaustive swimming time. The effects of blueberry polysaccharides on exercise endurance of mice are shown in Fig. (1).

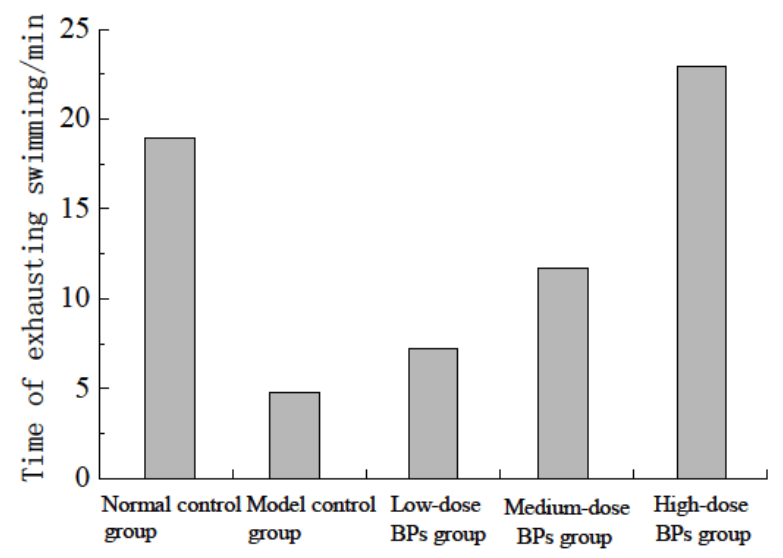

Fig. (1). Result of exhaustive swim of aged mice.

\subsection{Effects of BPs on Exhaustive Swimming Time of Aged Mice}

The time of continuous movement until exhausting and the survival time under the hypoxia condition can reflect the body's endurance, which is the relatively objective index to evaluate fatigue state. Methods for building aging animal models at pre-sent mainly include injection of D-galactose, ozone damage, thymus removal, asing of older animals, etc. As shown in Fig. (1), the exhaustive swimming time of mice in normal control group and different BPs dose groups are longer than that of the mice in the model control group. The exhaustive swimming time of the BPs low dose group has no significant difference $(\mathrm{P}>0.05)$ compared with the model control group. And the exhaustive swimming time of the rest groups is significantly longer than that of the model control 
Table 1. Effect of BPs on BUN and BLA of Aged Mice.

\begin{tabular}{|c|c|c|c|}
\hline Group & Dose(mg/kg·d) & BUN content /(m mol/L) & BLA content (mg/l00ML) \\
\hline \hline Normal control group & 100 normal saline & $13.26 \pm 1.78$ & $14.02 \pm 0.66$ \\
\hline Model control group & 0 & $15.32 \pm 2.05$ & $19.76 \pm 1.53$ \\
\hline Low-dose BPs group & 200 & $13.75 \pm 1.96$ & $18.01 \pm 0.94$ \\
\hline Medium-dose BPs group & 300 & $13.70 \pm 2.36$ & $15.97 \pm 0.27$ \\
\hline High-dose BPs group & 500 & $13.62 \pm 1.84$ & $14.15 \pm 0.99$ \\
\hline
\end{tabular}

Table 2. Effect of BPs on HG and MG of Aged Mice.

\begin{tabular}{|c|c|c|c|}
\hline Group & Dose(mg/kg·d) & HG content /(mg/g) & MG content (mg/g) \\
\hline \hline Normal control group & 100 normal saline & $30.28 \pm 4.87$ & $1.80 \pm 0.21$ \\
\hline Model control group & 0 & $21.16 \pm 3.16$ & $0.95 \pm 0.08$ \\
\hline Low-dose BPs group & 200 & $34.15 \pm 4.88$ & $1.22 \pm 0.07$ \\
\hline Medium-dose BPs group & 300 & $36.13 \pm 3.91$ & $1.57 \pm 0.17$ \\
\hline High-dose BPs group & 500 & $35.59 \pm 4.69$ & $1.81 \pm 0.10$ \\
\hline
\end{tabular}

group $(\mathrm{P}<0.05)$. The experimental results show that the exhaustive swimming time of the mice and the BPs dose are positively correlated, showing that blueberry polysaccharides can enhance the movement endurance of mice and have the anti-fatigue function. The exhaustive swimming time of mice in the BPs high-dose group is significantly longer than that of the mice in the BPs low-dose group $(\mathrm{P}<0.05)$. Results show that blueberry polysaccharides can significantly improve the exercise endurance of the aged mice and delay fatigue.

\subsection{Effect of BPs on Related Physiological Indexes Like BUN and BLA of Aged Mice}

After the experimental group rats were filled and fed with blueberry polysaccharides for 15 days, the results of BUN and BLA are shown in Table 1. The results show that the content of BUN of the dose group is lower than that of the control group, and the difference $(\mathrm{P}<0.01)$ is extremely significant, suggesting the blueberry polysaccharides have lower BUN.

When a large amount of glycogen is consumed, biological activity is reduced, resulting in the slow action and tiredness of body. Therefore, it is helpful to improve the body's endurance by reserving the original amount of nitrogen compounds conducive to reducing protein and sugar catabolism. Lack of oxygen supply relatively caused by strenuous exercise of body will increase metabolites of lactic acid etc., leading to drop of muscle contraction efficiency. The changing rule of the BLA content also appears similar. Compared with the model control group, the content of lowdose, medium-dose and high-dose BPs groups of BLA is reduced significantly $(\mathrm{P}<0.05)$.

\subsection{Determination Results of Liver Glycogen and Muscle Glycogen}

It can be seen from Table 2 that after the rats of all experimental groups are fed with blueberry polysaccharides 15 for days, liver glycogen content is increased significantly compared with the control group, and the difference $(\mathrm{P}<0.01)$ is extremely significant.

Table 2 shows that the HG content of different BPs dose groups is higher than that of the normal control group. Compared with the normal control group, the HG content in three BPs dose groups is significantly increased $(\mathrm{P}<0.05)$. There is evidence that after high intensity exercise for $2 \mathrm{~h}, \mathrm{MG}$ is almost exhausted, and hydrolysis of the HG is increased to maintain the stability of blood sugar as MG is reduced gradually. It can be seen that BPs can effectively enhance the capacity of continuous movement of the mice.

Numerous studies have found that $[5,6]$ the muscle fatigue caused by high-strength exercise within short time, is because ATP as the energy storage matter in muscle are in short supply. When ATP decreases to a certain level, MG will degrade to synthesize ATP, following the accumulation of lactic acid in the process, which in turn causes the rise of concentration of hydrogen ions in the body and drop of muscle $\mathrm{pH}$, and the muscle contraction ability will decrease eventually. The experiment confirms that the HG content of the BPs low-dose group, BPs medium-dose group, and BPs high-dose group is significantly higher than that of the normal control group $(\mathrm{P}<0.05)$, suggesting that BPs could increase the reserve of glycogen and provide the body with energy supplies. Hence, BPs have effects of relieving physical fatigue and enhancing the exercise endurance.

\subsection{Determination Results of MDA and Enzymes Dehy- drogenase Activity}

The experimental group rats were fed with blueberry polysaccharides for 15 days, and test results of MDA, SOD and LDH activities are shown in Table 3.

The results show that the lactate dehydrogenase activity of each BPs dose group is higher than that of the normal con- 
Table 3. Effect of BPs on MDA, SOD and LDH of Aged Mice.

\begin{tabular}{|c|c|c|c|c|}
\hline Group & Dose(mg/kg·d) & LDH vitality/(U/mL) & SOD vitality/(U/mL) & MDA content (mg/g) \\
\hline \hline Normal control group & 100 normal saline & $597.55 . \pm 42.15$ & $302.32 \pm 19.32 \mathrm{a}$ & $22.24 \pm 1.10$ \\
\hline Model control group & 0 & $539.55 . \pm 37.30$ & $250.01 \pm 13.74 \mathrm{~d}$ & $26.35 \pm 1.58 \mathrm{a}$ \\
\hline Low-dose BPs group & 200 & $585.09 . \pm 42.66$ & $311.27 \pm 24.47 \mathrm{a}$ & $25.40 \pm 1.32 \mathrm{ab}$ \\
\hline Medium-dose BPs group & 300 & $741.24 . \pm 76.62$ & $338.65 \pm 19.00 \mathrm{~b}$ & $23.29 \pm 1.04$ \\
\hline High-dose BPs group & 500 & $1027.61 \pm 98.84$ & $364.07 \pm 21.14 \mathrm{c}$ & $22.33 \pm 1.02 \mathrm{c}$ \\
\hline
\end{tabular}

trol group. The difference between the normal control group and the BPs medium-dose group is significant $(\mathrm{P}<0.05)$, and that between the normal control group and the BPs high-dose group is particularly significant $(\mathrm{P}<0.01)$, suggesting that the blueberry polysaccharides could increase the activity of MDA, SOD and LDH. Compared with the serum SOD activity of the model control group, that of the BPs low-dose, medium dose and high-dose groups has increased by $24.5 \%$, $35.4 \%$ and $45.6 \%$ respectively $(\mathrm{P}<0.05)$, suggesting that the regulation effect of BPs on serum SOD is very obvious. In addition, the serum LDH activity is also significantly increased with the increase of the BPs dose, but compared with other BP groups, the BPs high-dose group has no significant effect of serum LDH activity, which reflects the effect of BPs on the activity of LDH. The above results show that the BPs have good antioxidant effect on the body and it can not only significantly increase the antioxidant activity of the enzyme system in the body, but also effectively remove free radicals and prevent cell membrane lipid peroxidation, which is perhaps one of the key factors contributing to the antifatigue function of the BPs.

\section{CONCLUSION}

Fatigue refers to the temporary reduction of the ability to work due to change of the body function caused by excessive exercise; the exercise endurance is the ability to resist fatigue and strengthen the strong macro performance. The premise of the exercise endurance of the mice is increasing enough energy reserves, and the more the energy is supplied, the higher the endurance will be. BPs can reduce the content of BLA and BUN and increase the content of HG and MG in different degrees, and these changes may be the important material base of their roles in expressing anti-fatigue functions. Based on this, the time of exercise endurance of BPs high-dose group is longer than that of the normal control group by $14.3 \%(\mathrm{P}<0.05)$, the SOD and LDH activity of the mice in the BPs high-dose group is also effectively increased, and the content of BUN, BLA and MDA in mice is significantly reduced after strenuous exercise, which shows that the ability to remove free radicals is high by means of the high dose of BPs. Blueberry polysaccharides can increase the capacity of aerobic metabolism and exercise endurance of the mice. After completing the same exercise load, the BPs dosing group mice have relatively lower blood lactic acid content than that of the control group, thus able to resist the influence from exercise fatigue.

\section{CONFLICT OF INTEREST}

The author confirms that the content of this article has no conflict of interest.

\section{ACKNOWLEDGEMENTS}

This work is supported by the Key Project of Guangxi Social Sciences, China (No.gxsk201424), the Education Science Fund of the Education Department of Guangxi, China (No.2014JGA268), and Guangxi Office for Education Sciences Planning, China (No.2013C108).

\section{REFERENCES}

[1] A.M. Duquette and D.M. Andrews, "Tibialis anterior muscle fatigue leads to changes in tibial axial acceleration after impact when ankle dorsiflexion angles are visually controlled", Hum. Movem. Sci., vol. 29, no. 4, pp. 567-577, 2010.

[2] L. Yingchang and M. Xianjun, "Influence of main material content and dealing method in blueberries on anthocyanin", J. Food Sci., vol. 24, no. 5, pp. 163-164, 2008.

[3] N.J. Miller, J. Sampson and P. Candelasi, "Antioxidant activities of carotenes and xanthophylls", FEBS Lett., vol. 384, pp. 240-242, 1996.

[4] A.D. Ray, S. Udhoji and T.L. Mashtare, "A combined inspiratory and expiratory muscle training program improves respiratory muscle strength and fatigue in multiple sclerosis", Arch. Phys. Med. Rehabilit., vol. 94, no. 10, pp. 1964-1970, 2013.

[5] M.G. Sevag, D.B. Lackman and J. Smolens, "The isolation of components of streptococcal nucleoproteins in serologically active form”, J. Biolog. Chem., vol. 124, pp. 425, 1938.

[6] T. Wei, Y. Keqiang and L. Yanyan, "Anti-fatigue activity of polysaccharides extract from Radix rehmanniae Preparation", Int. J. Biolog. Macromol., vol. 50, no. 1, pp. 59-62, 2012.

\footnotetext{
Received: May 26, 2015

Revised: July 14, 2015

Accepted: August 10, 2015

(C) Bai Bo; Licensee Bentham Open.

This is an open access articles licensed under the terms of the Creative Commons Attribution-Non-Commercial 4.0 International Public License (CC BY-NC 4.0) (https://creativecommons.org/licenses/by-nc/4.0/legalcode), which permits unrestricted, non-commercial use, distribution and reproduction in any medium, provided that the work is properly cited.
} 